\title{
Engaging basic scientists in translational research
}

\author{
Anne M Deschamps and Charis Eng ${ }^{1}$
}

Federation of American Societies for Experimental Biology, 9650 Rockville Pike, Bethesda, Maryland 20814, USA

${ }^{1}$ Cleveland Clinic Genomic Medicine Institute, 9500 Euclid Avenue, Mailstop NE-50 (Rm NE5-314), Cleveland, Ohio 44195, USA

(Correspondence should be addressed to A M Deschamps; Email: adeschamps@faseb.org)

\section{Introduction}

Basic scientists play an essential role in improving human health. Their discoveries of biological processes and disease progression lay the groundwork for the development of new therapies, diagnostics, and devices. However, the pace at which these discoveries are translated into new medical interventions has been a subject of much concern (Butler 2008). The creation of the Clinical and Translational Science Award (CTSA) program in the USA in 2006, the establishment of the Cures Acceleration Network funded by the US Congress in 2011, and the formation of the National Center for Advancing Translational Sciences (NCATS) in 2012 signal the US federal government's commitment to translating discoveries made at the bench into public health improvements (Collins 2011, http://ncats.nih.gov/ (accessed 4/2/2012)). The UK's Medical Research Council doubled its biomedical research budget in fiscal year 2010, with the lion's share going to translational research. Continental Europe already has several CTSA-like programs. Yet despite the attention that translational research has received, there has been less focus on increasing the involvement of basic scientists in the translational research enterprise.

In March 2011, the Federation of American Societies for Experimental Biology (FASEB) sought to do just that. With the generous support of Howard Hughes Medical Institute, Doris Duke Charitable Foundation, the United States Department of Veterans Affairs, Burroughs Welcome Fund, and Merck, Sharp \& Dohme, FASEB organized a symposium that brought together scientists, research institution leadership, private and public funding organizations, scientific societies, and scientific publishers in an effort to identify ways these groups could facilitate the participation of basic scientists in translational research. The resulting report, 'Engaging Basic Scientists in Translational Research: Identifying Opportunities, Overcoming Obstacles', addresses actions that each of these groups can take to promote interest, education, and training in translational science; facilitate access to translational research collaborations and resources; recognize and reward translational scholarship; and enhance funding strategies for translational science (Hobin et al. 2012; www.faseb.org/Portals/0/PDFs/opa/TranslationalReportFINAL.pdf)

\section{Overcoming obstacles and identifying opportunities}

\section{Obstacle: lack of translational research education and training}

There is a lack of didactic training and experiential learning opportunities for basic scientists all along the research continuum - from graduate trainee though established principal investigator. According to symposium speaker, Mary C (Cindy) Farach-Carson, PhD, Vice Provost for Translational Bioscience at Rice University, today's graduate students have less contact with basic concepts of medicine than those in the past. They also have limited opportunities to interact with clinicians and patient populations. Indeed, this was the case for Michael Dyer, PhD, Director of the Division of Developmental Biology at St Jude Children's Research Hospital. During his 5 years at a major medical center, he never once met a clinician, patient, or patient's family. As a junior faculty member at St Jude's, however, clinicians who treated retinoblastoma patients invited him to the clinic. He eagerly accepted the opportunity and as a result shifted his program to one with a strong translational focus in which he developed new animal models to study disease and 
tested new therapeutic regimens for retinoblastoma treatment.

\section{Recommendations}

Such encounters like the one Dr Dyer experienced may not be as likely to emerge at other institutions, but universities can do things to help facilitate interactions between basic scientists and clinicians and encourage translational research training. Meeting participants recommended that institutions incorporate didactic coursework such as pathobiology and pathophysiology into graduate education, provide case-based learning opportunities, organize seminars that cover a variety of disease topics, and integrate clinical issues into the curricula of basic science training programs. Doing so would provide a foundation for understanding human health and disease processes. However, opportunities to develop skills in translational research should not be limited to only trainees. Institutions should allow established investigators time to participate in clinical rounds and/or take mini-sabbaticals to acquire clinical experience that would help to put basic research in context.

Professional societies also have a role to play in promoting interest and education in translational science. Societies should highlight translational research at meetings, establish networking opportunities to identify mentors, and offer workshops that provide practical tools for establishing translational research programs. Facilitating the establishment of summer research experiences for high school and undergraduate students would provide exposure to translational research early in one's career when they might more readily embrace it as a career path.

\section{Obstacle: limited access to translational research collaborations and resources}

Conference participants indicated that the cultural dissimilarities between basic and clinical scientists are a significant barrier to engaging in translational research. Basic and clinical investigators may speak different 'languages', have different research approaches, and even value their work differently. For example, basic scientists may see their research, which is focused on hypothesis testing, as more rigorous, while clinical scientists may feel that their goal-directed research is more relevant to human health. These differences oftentimes inhibit collaborations between the two groups.

Translational research involving human subjects may involve myriad administrative and regulatory requirements such as Institution Review Board protocol approvals, clinical trials registration, and reporting to data safety monitoring boards, with which basic scientists do not typically deal. Working with industry presents another level of complexity, including the development of data use and technology transfer agreements. Unfamiliarity with these requirements and the lack of resources and expertise to assist with these hurdles can dissuade basic scientists from engaging in translational research.

\section{Recommendations}

It is vital for institutions, funding agencies, individuals, and professional societies to facilitate interaction and collaboration among basic scientists, clinical scientists, and clinicians and provide access to the necessary research resources. At a minimum, providing opportunities for interaction will help to cultivate a better understanding of each other's work and the value they each bring to the enterprise, and ideally, these interactions will spur partnerships that lead to research advances. Developing funding mechanisms that encourage interdisciplinary partnerships; creating databases to identify collaborators, resources, and expertise; establishing centers that can provide research resources; and establishing networking events will all enable the participation of basic scientists in translational research.

\section{Obstacle: lack of appropriate recognition and reward for translational research}

Whereas basic science tends to be an independent endeavor where researchers carry out discipline-specific, hypothesis-driven studies, translational research is more likely to take the form of a large, collaborative, interdisciplinary, goal-directed project that can take a long time to conduct, particularly if it involves patients. The current tenure and promotions system within academia tends to reward the former and is not optimal for basic scientists pursuing translational research. Moreover, scientists can face challenges publishing translational research because, like basic science tenure committees, editorial boards and reviewers may place a higher value on hypothesis-driven basic science.

\section{Recommendations}

Investigators interested in pursuing translational science have a responsibility for understanding the tenure and promotion process at their institutions, negotiating the terms of their appointments, and defining the intellectual space in which they are going to be leaders. In addition, investigators should seek reviewers who understand and value the 
contributions that they make to translational research when soliciting tenure review letters.

However, institutions also have a role to play. They should consider revising their tenure and promotion polices to ensure that their investigators are recognized for their work on research teams and for the contributions they make outside of their disciplines. Institutions should recognize that evaluation of the investigator's scholarship may require review by those with diverse expertise. They should also consider the impact of a basic investigator's work - not just the quantity of publications - when evaluating their publication record. A number of institutions, including the University of Southern California, have put together models for how academic departments and committees can weigh interdisciplinary research and collaborative scholarship when making tenure and promotion decisions (http://www.usc.edu/academe/faculty/private/1011/UCAPT_Manual_Jan_2011_for_posting. pdf (accessed 4/2/2012)).

Because translational research papers can include many authors, publishers and editors should ensure that the roles of authors are clearly articulated in publications. Moreover, journals should publish editorials and commentaries that highlight the potential contributions a basic research finding might have to a particular disease state.

\section{Obstacle: limited funding strategies for translational science}

In spite of national efforts to promote translational research, some conference participants lamented the fact that they were unable to acquire sufficient funding to execute a translational research project. They also noted that study sections evaluating grant applications lacked the expertise to review translational protocols.

\section{Recommendations}

To address the challenges of acquiring translational research funding, the report recommends that funding organizations recognize studies aimed at open-ended inquiry and fund projects that engage investigators across disciplines. In addition, there should be mechanisms in place to provide supplemental funding to basic research grants allowing investigators to extend research findings in the translational research domain and for clinical investigators to conduct laboratory work based on clinical findings. Continued support for translational research infrastructure, commercialization centers, and core facilities would all facilitate the participation in translational research by basic scientists. Furthermore, grant review panels should be composed so that they have the appropriate expertise to review translational projects fairly. This may include creating additional translational research study sections and/or ensuring that translational researchers are among the scientists represented in each study section.

\section{Conclusion}

Creating an environment that encourages and enables basic researchers to pursue the translational potential of their work will be an important step in improving human health and treating disease. The recommendations and examples contained within the report provide a framework to enable each institution, individual, scientific society, publisher, or funding agency to tailor their policies and programs in order to achieve this goal.

\section{References}

Butler D 2008 Translational research: crossing the valley of death. Nature 453 840-842. (doi:10.1038/453840a)

Collins FS 2011 Reengineering translational science: the time is right. Science Translational Medicine 3 90 cm17. (doi:10.1126/scitranslmed.3002747)

Hobin JA, Deschamps AM, Bockman R, Cohen S, Dechow P, Eng C, Galey W, Morris M, Prabhakar S, Raj U, et al. 2012 Engaging basic scientists in translational research: identifying opportunities, overcoming obstacles. Journal of Translational Medicine 10 72. (doi:10.1186/1479. 5876-10-72) 